\title{
High-purity crystalline silicon gels
}

\section{Samantha L. Flores-López ${ }^{1}$, Lucía dos Santos-Gómez², Natalia Rey-Raap², Santiago García-Granda*2, Ana Arenillas ${ }^{1}$}

\author{
${ }^{1}$ Instituto de Ciencia y Tecnología del Carbono, INCAR-CSIC, 33011-Oviedo, Spain \\ ${ }^{2}$ Department of Physical and Analytical Chemistry, University of Oviedo - CINN-CSIC, 33006-Oviedo, Spain \\ sgg@uniovi.es
}

In the present investigation, silica gels have been synthesized via sol-gel method under microwave radiation. For that, precursor solutions were prepared using tetraethylorthosilicate (TEOS) as the silica precursor, varying the molar ratios of water and ethanol to it. $\mathrm{HCl}$ was added before heating to perform acid gelation, while $\mathrm{NH}_{3}(2 \mathrm{M})$ was added after gelation to promote polycondensation and Ostwald ripening reactions during aging. The use of microwave radiation under these conditions resulted in a favourable effect on the final structure of the polymeric network [1]. This approach makes it possible to obtain mesoporous silica gels in a short time, but amorphous in all cases (Fig. 1). The XRD pattern displayed the presence of a broad peak at $2 \theta=17-29^{\circ}$ that corresponds to the formation of amorphous silica according to JCPDS-card 96-900-1582.

The magnesio-thermal reduction process has already been reported as a useful way to convert silica into silicon in the presence of magnesium as a reduction agent [2,3]. Thus, our amorphous silica gels were mixed with $\mathrm{Mg}$ in a weight ratio of 1:1 and treated at 750 ${ }^{\circ} \mathrm{C}$ for $12 \mathrm{~h}$ under an inert atmosphere ( $\mathrm{Ar}, 300 \mathrm{~mL} / \mathrm{min}$ ). Many phases can be produced from the reduction process of $\mathrm{SiO}_{2}$ and $\mathrm{Mg}$, such as $\mathrm{MgO}$ and $\mathrm{Mg}_{2} \mathrm{Si}$. Thus, the reduced samples were subsequently washed with $\mathrm{HCl}(1 \mathrm{M})$ for 4 hours to eliminate the undesired secondary phases. The structural properties of the obtained silicon gels were analysed and measured by X-ray diffraction (XRD), Xray fluorescence (XRF) and high-resolution transmission electron microscopy (HR-TEM). Fig. 1 shows the XRD data of the final reduced silicon gel, illustrating the complete removal of $\mathrm{SiO}_{2}$, with only $\mathrm{Si}$ peaks remaining in the structure. The major diffraction peaks at $2 \theta=28.4^{\circ}, 47.4^{\circ}$ and $56.2^{\circ}$ are presented at (111), (202) and (131) planes, respectively, which can be attributed to high-purity silicon gel according to JCPDS-card 96-901-3109. Also, the absence of additional peaks indicates that no impurities are present in the structure.

AMORPHOUS SILICA GEL

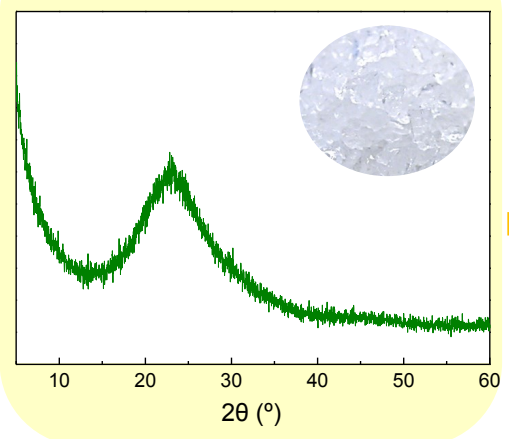

CRYSTALLINE SILICON GEL

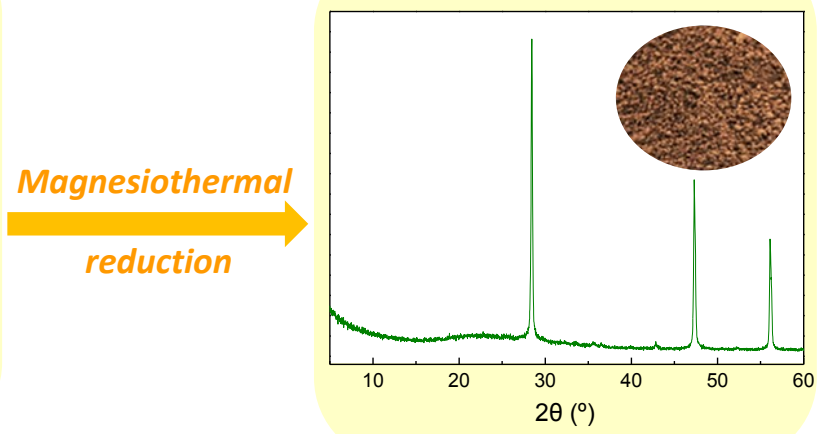

Figure 1. XRD data of a) silica gel before treatment and b) silicon gels after magnesio-thermal reduction.

[1] Flores-López, S.L., Villanueva, S.F., Montes-Morán, M.A., Cruz, G., Garrido, J.J. \& Arenillas, A. (2020). Colloids Surf. Physicochem. Eng. Asp. 604, 125248.

[2] Xing, A., Zhang, J., Bao, Z., Mei, Y., Gordin, A.S. \& Sandhage, K.H. (2013). Chem. Commun. 49, 6743.

[3] Jia, H., Gao, P., Yang, J., Wang, J., Nuli, Y. \& Yang, Z. (2011). Adv. Energy Mater. 1, 1036.

Keywords: Amorphous silica gel; Crystalline silicon gel; Magnesiothermal reduction; Microwave heating.

The authors thank Prof. José R. García, University of Oviedo, Spain, for his appreciated contribution. 